\title{
The cholesterol hypothesis and the value of intervention
}

\author{
W. VIRGIL BROWN, MD
}

The cholesterol hypothesis contends that hypercholesterolemia plays a major role in the etiology of coronary heart disease (CHD) and that interventions that lower serum cholesterol levels will lower incidence mortality. Prospective clinical trials carried out over nearly four decades have shown that elevated values of blood cholesterol, especially low density lipoprotein cholesterol, confer a much higher risk of the patient's later developing CHD. Not until recently, however, have scientific studies shown that interventions to reduce serum cholesterol levels are worthwhile for many patients and that routine identification of these patients is possible. Some of these important clinical studies, particularly the Lipid Research Clinics Coronary Primary Prevention Trial and the Cholesterol-Lowering

Atherosclerosis Study, are reviewed. Ongoing investigations concern the importance of other risk factors, such as high density lipoprotein cholesterol and some of the apolipoproteins.

The cholesterol hypothesis holds that elevated serum cholesterol levels are causally related to clinical disease and mortality stemming from atherosclerosis. It maintains, furthermore, that interventions that lower a patient's cholesterol level will also lower mortality attributable to coronary disease.

One of the major medical questions of our time has been the proof of the cholesterol hypothesis. Atherosclerosis and its sequelae of myocardial infarction, sudden cardiac death, stroke, heart failure, angina, intermittent claudication, and other phenomena impose enormous burdens of mortality and morbidity on individual patients and on society. If the cholesterol hypothesis were true, it would immediately be attractive to propose interventions aimed at reducing circulating levels of $\mathrm{LDL}$, in the hope of increasing both survival and the quality of patients' lives. The argument has been whether intervention will produce significant clinical benefit, and, if so, whether those patients who will profit from such treatment can be identified.

A great deal of effort in attempting to answer these questions has been expended in recent years. It now appears that lowering serum cholesterol levels is worthwhile indeed. Even so, much remains to be understood if we are to direct our treatment to patients who need it, yet avoid wasting resources and raising anxiety in patients who do not need it.

However, a benchmark of sorts has been reachedrecently with the establishment of guidelines for detecting, evaluating, and treating hypercholesterolemia in adults by the National Cholesterol Education Program. ${ }^{1,2}$ The approach suggested is complex. However, the problems at which it is directed are complex also. This paper will review some of the clinical studies important to the development of the report, then suggest some future directions for intervention.

\section{Early clinical experience}

The relationship between serum cholesterol and the risk of morbidity and mortality from heart disease has been demonstrated in a number of studies. The Framingham trial ${ }^{3}$ is doubtless the best known, and was one of the earliest to enroll enough subjects to obtain convincing results. In that study, in men 30 to 49 years old followed for 8 years, there was more than a fourfold increase in risk between cholesterol levels of 200 and $260 \mathrm{mg} / \mathrm{dL}$. The largest study relating blood cholesterol to risk was generated by following the men (35-59) who were screened for the multiple risk factor intervention trial. ${ }^{4}$ The risk of coronary heart disease was fourfold greater for a man with cholesterol $300 \mathrm{mg}$./ $\mathrm{dL}$, as opposed to those with $200 \mathrm{mg} / \mathrm{dL}$. Subjects at the upper end of this range had almost twice the average risk of heart disease, whereas subjects at the lower end had about a third of the average risk.

The findings spurred research interest in the possible value of lowering blood cholesterol levels. It was hoped that risk would be lowered in proportion to the reduction in blood cholesterol values so that we would, in essence, be able to move individual patients back down the curve. Unfortu- 


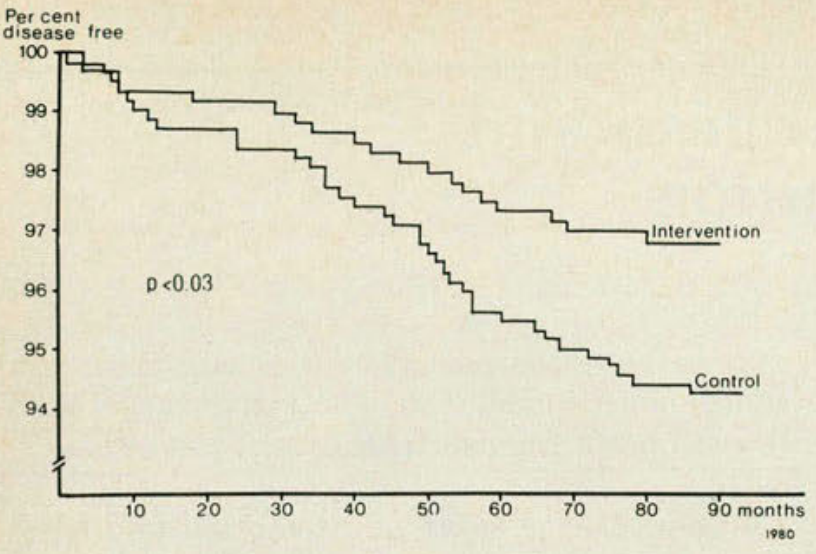

Fig 1. Life table analysis of CHD (fatal and nonfatal myocardial infarction and sudden death) in intervention and control groups. Reprinted from Lancet (1981;2:130!-1310), Copyright 1981, Lancet.

nately, a number of the earlier studies of intervention suffered from procedural flaws that blurred the value of their conclusions. Nonetheless, they convincingly pointed the way for later investigators.

Among these pioneer trials was one conducted by Dayton's group 5 at the Wadsworth Veteran Administration Hospital. They randomly assigned 846 veterans aged 54 to 88 years to one of two kitchens. The first kitchen fed the standard VA diet, which was high in saturated fat and cholesterol$20 \%$ of calories from saturated fat, and about 650 $\mathrm{mg}$ /day of cholesterol. The second kitchen provided a diet similar to that recommended by the American Heart Association, but with added emphasis on polyunsaturated fats. Saturated fats were reduced to $10 \%$ and cholesterol to $365 \mathrm{mg} /$ day.

The subjects were followed for 8 years, during which time the group fed the modified diet experienced a sustained drop in cholesterol levels of about $13 \%$. There was a trend toward fewer cardiovascular events in the latter group, but the difference did not reach statistical significance. When the data from younger patients were analyzed separately, however, the trend was much stronger. The subjects in this study were considerably older than those enrolled in subsequent trials of this sort. There is little doubt that had the patient cohort been younger and the study a year or two longer, the data would have achieved significance. In any event, the outcome pointed the way for more definitive trials.

A trial reported in 1981 from the Oslo study group 6 examined the value of lowering serum triglyceride and cholesterol levels through dietary intervention, as well as the effect of reductions in smoking. The subjects were 1,232 CHD-free men whose serum cholesterol levels averaged $325 \mathrm{mg} /$ $\mathrm{dL}$. Those in the intervention group on the reducedcholesterol diet showed a sustained serum-cholesterol drop of about $13 \%$, which was significantly lower than the controls. This was the same reduction as was seen in the VA cohort, but it was achieved in a free-living patient population having a much greater likelihood of noncompliance with the diet. Counseling efforts resulted in a reduction in cigarette smoking of about $50 \%$ in the intervention group.

In this study there was a highly significant reduction in fatal and nonfatal myocardial infarction and sudden death (Fig 1). Almost $97 \%$ of the intervention group was free of CHD after 90 months, compared with $94 \%$ of the controls-a nearly $50 \%$ difference in the incidence of disease $(P<.03)$. The difficulty is that two risk factors were manipulated simultaneously; thus, the value of lowering cholesterol alone was not proved conclusively. However, in terms of affecting the risk, the reduction in cigarette smoking was estimated as being relatively smaller as compared to the effect of reduction in cholesterol, so the latter in all likelihood would have produced a significant reduction in CHD even if it had been evaluated independently.

\section{Lipid Research Clinics Trial}

The Lipid Research Clinics Coronary Primary Prevention Trial (LRC-CPPT) ${ }^{7-9}$ was a much larger intervention study carried out in the United States, beginning around 1972. It involved a collaboration between the National Institutes of Health $(\mathrm{NIH})$, 12 university-based lipid clinics, and three other centers. This was the first of the major trials to provide incontrovertible evidence that cholesterol reduction results in a reduced risk of CHD.

A total of 3,806 middle-aged, hypercholesterolemic men with no signs or symptoms of coronary disease were enrolled. ${ }^{10}$ The principal intervention agent was cholestyramine resin. This drug had been shown in clinical trials to lower serum cholesterol dependably by $20 \%$ to $25 \%$. A target of a $20 \%$ reduction was chosen in this study. Approximately half of the subjects were assigned to an experimental group receiving cholestyramine and dietary treatment and half to a control group treated with placebo and diet. All patients were monitored for at least 7 years for the development of CHD.

An important difference in this trial was the focus on changes in LDL cholesterol rather than simply total serum cholesterol. The latter, however, has a surrogate parameter for LDL because the associated risks are virtually identical since nearly all of the risk from total cholesterol is due to the 


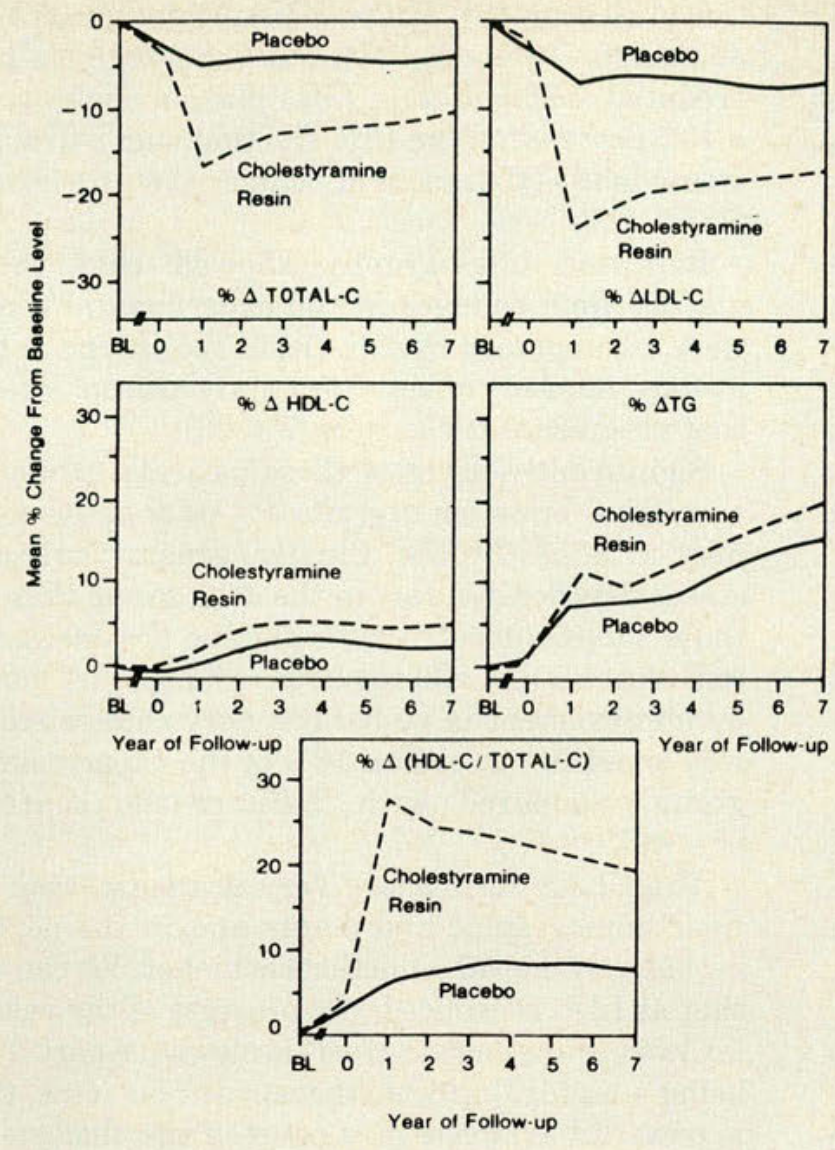

Fig 2. Mean yearly plasma lipid levels for cholestyramine- and placebo-treated men. On abscissa, BL represents baseline (prediet) period and 0 years represents 3-month interval between initiation of Lipid Research Clinics diet and study medication. Year 1 is average of visits 7 through 13, and each year thereafter represents average of six visits. $\Delta$ indicates change from baseline level; TOTAL-C, plasma total cholesterol levels; $L D L-C$, low density lipoprotein cholesterol levels; TG, triglyceride levels; and HDL-C, high density lipoprotein cholesterol. Reprinted from The Journal of the American Medical Association (1984;245:351-364), Copyright 1984, American Medical Association.

LDL fraction. Persons with triglyceride levels above $300 \mathrm{mg} / \mathrm{dL}$, that is, those with elevated chylomicrons and very low density lipoprotein, were excluded. The high density lipoprotein (HDL) level was not considered in choosing study subjects.

Candidates for the study had to demonstrate resistance to diet therapy. Those who started with LDL values above the 95th percentile for the 40 year-old age group and whose $\mathrm{LDL}$ values remained within the 90 th percentile $(>175 \mathrm{mg} / \mathrm{dL})$ despite a low-cholesterol diet were admitted to the study.

In the control group, diet alone resulted in a downward drift from the baseline total cholesterol and LDL cholesterol means of about $5 \%$ by the end of the first year (Fig 2). A $16 \%$ to $17 \%$ reduction in total cholesterol level was seen in the drug group at this time, but the reduction in LDL cholesterol level approached $24 \%$, because of the selectivity of cholestyramine for this fraction. Surprisingly, in the drug group the HDL fraction rose about 5\% after the 7 years, a rise that, combined with the drop in total cholesterol, produced a sharp increase in the HDL cholesterol/total cholesterol ratio.
Over the course of the trial, both total cholesterol and LDL cholesterol values drifted upward once more, principally because of falling patient compliance with the drug regimen. At the end of the study, the drug group had a mean LDL cholesterol level only $11 \%$ lower than the group receiving diet therapy alone. There was considerable concern at first that this very expensive effort had failed in its objectives. However, when the outcome was examined, the experimental group had achieved very nearly a $20 \%$ reduction in the incidence of myocardial infarction and sudden death. This was clear-cut evidence that lowering LDL cholesterol levels lowers the risk of morbidity and mortality. For a small reduction of serum cholesterol level in hypercholesterolemic men, a very marked impact on disease events was obtained. It is likely that some benefit also was dervied from the increases in HDL levels.

\section{Measure of risk reduction}

Out of this trial came the familiar extrapolation that for every $1 \%$ reduction that is achieved in to- 
tal serum cholesterol, the patient will experience a $2 \%$ reduction in CHD events. However, it should be remembered that the subjects enrolled had LDL cholesterol levels over the 90 th percentile. The same dramatic responses may not occur in patients with more moderate LDL cholesterol levels.

At first glance, the reduction in risk achieved in the LRC-CPPT trial appears to match the outcome reduction in coronary events as one moves back down the Framinghar.. risk curve. If so, the implication would be that patients treated after many years of hypercholesterolemia would almost immediately achieve the same risk they would have experienced had they never had higher levels of serum cholesterol, a conclusion that is suspect.

According to Peto, however, the Framingham curve should in fact be much steeper. As drawn, it was based on a single cholesterol measurement in each subject. There are inevitable analytical errors in cholesterol determinations. A statistical consequence of plotting error-prone single measurements is a tendency for the population to spread out artifactually on either side of the mean. If repeated measurements are averaged and plotted, on the other hand, this regression to the mean is reduced.

The true range of serum cholesterol levels against which the incidence of disease events must be plotted is, therefore, narrower than shown in the Framingham risk curve. When the curve is corrected to allow for regression to the mean, the increase in risk of $\mathrm{CHD}$ events for each $1 \%$ rise in cholesterol levels at the higher end of the cholesterol range is closer to $3 \%$ than $2 \%$. Thus, lowering serum cholesterol levels through intervention does not appear to erase all the risk from previous exposure to the higher levels. On the other hand, there is even greater incentive for intervention to prevent continued increases in LDL cholesterol values.

\section{Cholesterol-Lowering Atherosclerosis Study}

Waiting for CHD to develop is at best a crude way of measuring patient's responses to serum cholesterol reduction, and recent investigations have used more sophisticated and direct methods. In the Cholesterol-Lowering Atherosclerosis Study (CLAS) reported a year ago, for example, Blankenhorn and colleagues $^{12}$ studied 162 nonsmoking men 40 to 59 years of age who had previously undergone coronary bypass surgery and who had moderate to high levels of serum cholesterol.

In this trial, cardiac catheterization was performed to document existing vascular lesion status and to establish baseline status. Subjects in the experimental group next were treated aggressively to lower their cholesterol levels, using a combination of diet and two drugs, colestipol hydrochloride and niacin. After only two years this regimen had produced a $26 \%$ drop in total plasma cholesterol, a $43 \%$ decrease in the LDL fraction, and a $37 \%$ increase in the HDL fraction, compared with controls (Table $1^{12}$ ).

Repeated angiography showed that these marked lipid changes in the experimental group were accompanied by significant reductions in the average numbers of native coronary arterial lesions that progressed per subject $(P<.03)$.

Significantly fewer of these patients exhibited new lesion formation in grafts $(P<.04$ or native coronary arteries $(P<.03)$. Finally, general coronary status deteriorated less in the drug group than in those subjects treated with placebo $(P<.001)$. Actual regression of atherosclerotic changes, as shown by improvement in global coronary change score, was found in more than $16 \%$ of the experimental group, compared with $2.4 \%$ of the controls $(P=.002)$.

The CLAS results are very dramatic, both in their angiographic end points and in the degree of cholesterol level reductions achieved. We can see that as LDL is reduced, the progress of the vascular lesions of atherosclerosis is slowed. Apart from being a highly desirable therapeutic outcome, this is powerful evidence in support of the cholesterol hypothesis, if any more evidence is needed.

As for the cholesterol reductions, the average LDL cholesterol level achieved in the drug group was $97 \mathrm{mg} / \mathrm{dL}$, almost down to the level seen in vegetarians in this country, and well below the average of about $150 \mathrm{mg} / \mathrm{dL}$ for middle-aged American men. Clearly, therefore, aggressive intervention is capable of producing major decrements in total cholesterol and in the LDL fraction with beneficial effects on diseased arteries.

\section{Future interventions}

With clear evidence that LDL cholesterol reduction is both worthwhile and achievable, it is appropriate to look at other avenues of intervention in patients' lipid status that might affect their risk of suffering CHD events. Certainly, attempting to raise the levels of HDL cholesterol would have to be high on the list.

At the present time, we have no clear rationale for raising HDL other than that it is a changeable factor related to risk. However, the inverse relationship between HDL levels and CHD risk has been well documented in some 25 studies, a major one being the Framingham study itself. ${ }^{13-16}$ What we need to know is whether intervention will permit a patient to move down the curve toward lower 
TABLE 1. MEAN (SEM) BASELINE AND ON-TRIAL FASTING LIPID LEVELS BY TREATMENT GROUP.*

\begin{tabular}{|c|c|c|c|c|c|}
\hline \multirow{3}{*}{$\begin{array}{l}\text { Lipid or } \\
\text { lipoprotein }\end{array}$} & \multirow{3}{*}{$\begin{array}{l}\text { Treatment } \\
\text { group }\end{array}$} & \multicolumn{4}{|c|}{ Lipid level, mg/dL (mmol/L) } \\
\hline & & \multirow[b]{2}{*}{ Baseline } & \multirow[b]{2}{*}{ On-trial } & \multicolumn{2}{|l|}{ Changet } \\
\hline & & & & Difference & $\%$ \\
\hline Total & D & $246[4](6.35[0.09])$ & $180[3](4.65[0.09])$ & $-66[3] \div(-1.70[0.08])$ & $-26[1]$ \\
\hline cholesterol & $\mathrm{P}$ & $243[4](6.28[0.10])$ & $232[4](6.00[0.10])$ & $-11[2] \div(-0.28[0.06])$ & $-4[1]$ \\
\hline Triglycerides & $\begin{array}{l}\mathrm{D} \\
\mathrm{P}\end{array}$ & $\begin{array}{l}151[9](1.71[0.10]) \\
154[10](1.74[0.11])\end{array}$ & $\begin{array}{l}110[5](1.25[0.06]) \\
141[9](1.59[0.10])\end{array}$ & $\begin{array}{l}-41[6] \ddagger(-0.46[0.07]) \\
-13[4] \div(-1.15[0.04])\end{array}$ & $\begin{array}{r}-22[2] \\
-5[2]\end{array}$ \\
\hline $\begin{array}{l}\text { LDL } \\
\text { cholesterol }\end{array}$ & $\begin{array}{l}\mathrm{D} \\
\mathrm{P}\end{array}$ & $\begin{array}{l}171[3](4.42[0.09]) \\
169[3](4.36[0.09])\end{array}$ & $\begin{array}{r}97[3](2.51[0.07]) \\
160[3](4.13[0.08])\end{array}$ & $\begin{array}{r}-74[3] \ddagger(-1.91[0.08]) \\
-9[2] \neq(-0.23[0.06])\end{array}$ & $\begin{array}{r}-43[1] \\
-5[1]\end{array}$ \\
\hline $\begin{array}{l}\text { HDL } \\
\text { cholesterol }\end{array}$ & $\begin{array}{l}\mathrm{D} \\
\mathrm{P}\end{array}$ & $\begin{array}{l}44.6[1.0](1.15[0.03]) \\
43.7[1.0](1.13[0.03])\end{array}$ & $\begin{array}{l}60.8[1.4](1.57[0.04]) \\
44.4[0.9](1.15[0.02])\end{array}$ & $\begin{array}{r}16.2[0.9] \neq(0.42[0.02]) \\
0.7[0.6](0.02[0.01])\end{array}$ & $\begin{array}{r}37[2] \\
2[1]\end{array}$ \\
\hline $\begin{array}{l}\text { LDL/HDL } \\
\text { ratio }\end{array}$ & $\begin{array}{l}\mathrm{D} \\
\mathrm{P}\end{array}$ & $\begin{array}{l}4.0[0.1] \\
4.0[0.1]\end{array}$ & $\begin{array}{l}1.7[0.1] \\
3.7[0.1]\end{array}$ & $\begin{array}{l}-2.3[0.1] \ddagger \\
-0.3[0.1] \ddagger\end{array}$ & $\begin{array}{r}-57[1] \\
-6[2]\end{array}$ \\
\hline $\begin{array}{l}\text { *Eighty subjects } \\
\text { †Group differenc } \\
\text { †Significant at } P \\
\text { Reprinted from }\end{array}$ & $\begin{array}{l}\text { receiving dr } \\
\text { e significant } \\
\text { using a pair } \\
\text { ournal of the }\end{array}$ & $\begin{array}{l}\text {; } 82 \text {, placebo (P). } \\
<.001 \text { using the independ } \\
\text { cudent's } f \text { test against a tv } \\
\text { erican Medical Associatio }\end{array}$ & $\begin{array}{l}\text { udent's } t \text { test against a t } \\
\text { d alternative. } \\
; 257: 3233-3240 \text { ), Copyr }\end{array}$ & $\begin{array}{l}\text { d alternative. } \\
\text { 87, American Medical Ass }\end{array}$ & \\
\hline
\end{tabular}

risk. A study, ${ }^{16}$ has recently been completed in Helsinki using gemfibrozil, a drug that reliably produces about a $10 \%$ elevation in HDL cholesterol, while lowering LDL by about the same percentage. The trial enrolled approximately 4,000 men with primary dyslipidemia who had no evidence of $\mathrm{CHD}$, to determine whether raising their HDL levels would lead to the predicted reduction in CHD events. After 5 years, the drug group showed a $34 \%$ reduction in the incidence of $\mathrm{CHD}(P<.02)$, strongly suggesting that raising HDL with this agent is also effective in reducing risk.

A number of other risk factors are being investigated in addition to HDL, though not at such an advanced stage. Among these are the apolipoproteins A-I and B (apoA-1 and apoB). ApoA-I is the major component of HDL. There are a number of case-controlled studies in which apoA-I has been found to be much decreased in patients with $\mathrm{CHD}$, whereas apoB is elevated. ${ }^{18}$ Some investigators maintain that apoA-I and/or apoB measurements allow them to segregate subjects with $\mathrm{CHD}$ better than do measurements of cholesterol fractions. ${ }^{19-21}$ Further studies are needed to determine whether apoA-I, apoB, and possibly other apolipoproteins are independent risk factors for CHD. If so, we will have to determine whether they can be influenced to reduce risk.

However, we do not yet have the CHD incidence data for such parameters that we have for the cholesterol content of the lipoproteins. Thus we cannot establish risk of CHD or predict outcome by measuring apolipoprotein levels. Furthermore, we have very little idea of the clinical consequences of raising the level of apoA-I, for instance, or low- ering apoB, or if this can even be done reliably. Therefore it is premature to suggest using the apolipoproteins clinically. At present they are essentially research tools.

\section{Conclusion}

In light of the representative studies outlined here and many others, there can be little doubt that the cholesterol hypothesis holds true: Elevated serum cholesterol is linked to the etiology of atherosclerosis and the resulting CHD. Lowering of serum cholesterol retards the progression of atherosclerosis and may even reverse the process. And, the slowing or reversal of atherogenesis in turn reduces the risk of CHD.

Besides establishing the validity of the cholesterol hypothesis, these studies have gone a long way toward delineating the practical measures that should be taken in managing a patient with hypercholesterolemia. The $1985 \mathrm{NIH}$ consensus report on cholesterol and heart disease ${ }^{22}$ made a very strong statement that dealing effectively with hypercholesterolemia could reduce the rate of $\mathrm{CHD}$, at least in men.

In response to the consensus panel's mandate, $\mathrm{NIH}$ established the National Cholesterol Education Program. (This is a companion program to an earlier one dealing with hypertension.)

These resulting guidelines, which are summarized by $\mathrm{Voss}^{2}$ in this issue of JAOA (p 845) are complex, and their full implementation will take years. However, they represent the most ambitious attempt to guide health professionals in reducing the morbidity and mortality stemming from hypercholesterolemia. 
1. Report of the National Cholesterol Education Program expert panel on detection, evaluation, and treatment of high blood cholesterol in adults. Arch Int Med 1988;148:36-69.

2. Voss LWH: Detection, evaluation, and treatment of hypercholesterolemia in adults: Commentary on the National Cholesterol Education Program guidelines. JAOA 1988;88:845-853.

3. Castelli WP, Garrison RJ, Wilson PWF, et al: Incidence of coronary heart disease and lipoprotein cholesterol levels: The Framingham Study. JAMA 1986;256:2835-2838.

4. Kannel WB, Neaton JD, Wentworth D, et al: Overall and CHD mortality rates in relation to major risk factors in 325,348 men screened for the Multiple Risk Factors Intervention Trial. Am Heart $J$ 1986;112:825-836.

5. Dayton S, Pearce ML, Hashimoto S, et al: A controlled clinical trial of a diet high in unsaturated fat in preventing complications of atherosclerosis. Circulation 1969;40(suppl 2):1-63.

6. Hjermann I, Velve Byre K, Holme I, et al: Effect of diet and smoking intervention on the incidence of coronary heart disease: Report from the Oslo Study Group of a randomised trial in healthy men. Lancet 1981;2:1304-1310.

7. The Lipid Research Clinics Program: The Lipid Research Clinics Coronary Primary Prevention Trial results: I. Reduction in incidence of coronary heart disease. JAMA 1984;251:351-364.

8. The Lipid Research Clinics Program: The Lipid Research Clinics Coronary Primary Prevention Trial results: II. The relationship of reduction in incidence of coronary heart disease to cholesterol lowering. JAMA 1984;251:365-374

9. Rifkind BM: Lipid Research Clinics Coronary Primary Prevention Trial: Results and implications. Am J Cardiol 1984;54:30C-34C.

10. Levy RI, Fredrickson DS, Stone NJ, et al: Cholestyramine in type II hyperlipoproteinemia: A double-blind trial. Ann Intern Med 1973;79:5158.

11. Gordon T, Castelli WP, Hjortland MC, et al: High-density lipoprotein as a protective factor against coronary heart disease. Am $J$ Med 1977;62:707-14.

12. Blankenhorn DH, Nessim SA, Johnston RL, et al: Beneficial effects of combined colestipol-niacin therapy on coronary atherosclerosis and coronary venous bypass grafts. JAMA 1987;257:3233-3240.

13. Gordon T, Castelli WP, Hjortland MC, et al: High density lipopro- tein as a protective factor against coronary heart disease: The Framingham study. Am $J$ Med 1977;62:707-714.

14. Casteili WP, Garrison RJ, Wilson PWF, et al: Incidence of coronary heart disease and lipoprotein cholesterol levels: The Framingham study JAMA 1986;256:2835-2838.

15. Rhoads GG, Gulbrandsen CL, Kagan A: Serum lipoproteins and coronary heart disease in a population study of Hawaiian Japanese men. $N$ Engl J Med 1976;294:293-298.

16. Miller NE: The evidence for the antiatherogenicity of high density lipoprotein in man. Lipids 1978;13:914-919.

17. Frick MH, Elo O, Haapa K, et al: Helsinki heart study: Primaryprevention trial with gemfibrozil in middle-aged men with dyslipidemia. Safety of treatment, changes in risk factors, and incidence of coronary heart disease. $N$ Engl $J$ Med 1987;317:1237-1245.

18. Kwiterovich PO Jr, Sniderman AD: Atherosclerosis and apoproteins B and A-I. Prevent Med 1983;12:815-834.

19. Maciejko JJ, Holmes DR, Kottke BA, et al: Apolipoprotein A-I as a marker of angiographically assessed coronary-artery disease. $N$ Engl $J$ Med 1983;309:385-389.

20. Brunzell JD, Sniderman AD, Albers JJ, et al: Apoproteins B and A-I and coronary artery disease in humans. Atherosclerosis 1984;4:79. 83.

21. Teng LB, Thompson GR, Sniderman AD, et al: Composition and distribution of low density lipoprotein fractions in hyperapobetalipoproteinemia, normolipidemia, and familial hypercholesterolemia. Proc Natl Acad Sci 1983;80:6662-6666.

22. National Institutes of Health: Lowering blood cholesterol to prevent heart disease. JAMA 1985;153:2080-2086.

Dr. Brown is cochairman, Diet Treatment Subcommittee, National Cholesterol Education Program, and President and Chief Executive Officer, Medlantic Research Foundation, Washington, DC.

Reprints requests to Dr Brown, Medlantic Research Foundation, 108 Irving St NW, Washington, DC 20010. 